\title{
Use of Models in Reverse Thinking
}

\author{
Romans Vitkovskis \\ Latvian Education Foundation, Riga, Latvia \\ Uldis Heidingers \\ Riga State Technical School, Riga, Latvia; \\ Latvian Education Foundation, Riga, Latvia
}

\begin{abstract}
The fact that students tend to somewhat ignore the Science, Technology, Engineering, and Mathematics (STEM) subjects can be linked to their belief that the knowledge given in school on these subjects are hopelessly out of date, useless in everyday practice, and therefore, they lack motivation. This belief gets even stronger since the Internet is full with answers and ready-made solutions to handle all the question viewed during the study process. Comprehension of the subject and other things become insignificant if the subject itself is considered useless. For the subject not to be absolutely rejected, each course should include the basic philosophy, structure, and the themes currently viewed in the field as well as the necessary minimum to start getting acquainted with the topicalities of the subject. It is obvious that in a STEM subject (the study courses of STEM subjects have less or almost enough information to start getting acquainted with the topicalities) the comprehension is important. If it lacks, the student starts to dislike and reject the subject. This is also true in everyday life when encountering new concepts, relations, engineer technical solutions, etc., new things. Therefore, alongside building motivation, means of gaining comprehension must be found and knowledge must be used not only in the field of the given subject. For this purpose, reverse thinking is the useful as described in this paper. If someone has the ability to reduce matters of a subject to fit other things of other persons or things previously known to him/her, it is an example of only restricted reverse thinking. If the person has the ability to do so unintentionally in any situation, it can be called reverse thinking as we understand it. Practice of creation and usage of the models in a given subject + models in other subjects and demonstration how this approach is used universally and intentionally show that this approach let you understand a lot of new things, gradually habituates to unintentional use the models and helps to "understand the idea," and finally, leading to subconscious use of the models. Reverse thinking is one of phenomenons typical to the human brain and, as the eight years long practice shows (see http://www.goerudio.com), is actually possible. Students are able to create models that answer the question "why," but the created model basis allows inventing or re-discovering the same thing again. This already is an experimental result and is available in the public space (see http://www.goerudio.com), showing the student models for STEM subject topics.
\end{abstract}

Keywords: comprehension, model, subject, re-discover

Romans Vitkovskis, member of the board, Latvian Education Foundation.

Uldis Heidingers, teacher, Riga State Technical school; Latvian Education Foundation. 


\section{Reverse Thinking and How to Recognise It}

Reversible/reverse processes is a particular category, because it is impossible to reverse some processes (e.g., turn a roasted duck back into a living duck), but steam can condense and turn back into liquid water. Usually, with reverse action only approximate result is possible, because there can be objects that might not have taken part in the direct process (e.g., when integrating a function one cannot be sure if it is a derived function). Scientific discoveries and engineer technical solutions often are made thanks to the ability to see similarities in different areas and combine them, and they will include not only experimental data, but also thing created by other people. In order to do this, one must understand the results reached by others. If the aim is to create something new, one must be able to quickly understand things (concepts and connections) in other areas-how they were discovered and why they do not work in some other way (Cyriel, 2017). Reverse thinking as described in this paper is the ability to use ideas of one area in another on everyday level. It can be observed when looking how a person is capable to explain (mainly to himself/herself) things with models (i.e., matters from one given area as a part of another area). If one is capable of this not only in a professional area, but also in everyday life, he/she uses reverse thinking. If the person is capable of this only in a professional area, he/she uses restricted reverse thinking. One example of this could be an auto mechanic who is able to use a model and explain how a car suspension works, but we can see that he/she is not able to find a model that would explain how his/her washing machine works. If he/she had reverse thinking, he/she could use a model to explain how his/her washing machine works as well as why his/her relationship with wife is getting weaker. Reverse thinking can only be recognised if the person is able to create models practically in all situations in every area of his/her life.

\section{Need for Reverse Thinking}

Not very long ago, the skills and knowledge acquired in school and university were still useful for many years. Right now, both the skills and knowledge obtained through the learning process are out of date and useless in the labour market. We must either be able to justify the reasons for teaching all this or give up and declare it nonsense. The same is true with many trade subjects taught in school as from the moment the child enters the school all the equipment is already dated. In the outcome, the knowledge acquired thorough the learning process will be of no practical use. If we consider the knowledge to be information and comprehension, everything can be fixed by changing the emphasis in the learning process. During the learning process, comprehension and a way of gaining it by the use of models that have proved to be effective should be taught, as well as the reverse thinking with the use of models. Education does not have to provide knowledge on specific things, rather than teach how to gain new knowledge. It should teach how to learn. Here, the model method and the reverse thinking becomes one of the best ways of learning.

European Union (EU) project "GoScience" is one of the first steps to introduce the desire to learn something new in order for the students to feel more comfortable in everyday situations. The main focus of the "GoScience" project-comprehension is no miracle cure, but serves good enough for every student to feel comfortable in a given area. Life quality can be measured by how comfortable we feel and it affects all students. If the student feels comfortable, there are not and never will be problems with comprehending Science, Technology, Engineering, and Mathematics (STEM) or any other subjects. The knowledge is only valid until new knowledge takes its place by undermining, substantially extending, or rearranging it. This is a 
constant process of which we can see only the results.

Reverse thinking as we understand it roots the students attitude to things, that is, the ability to learn in the current world governs the students capability to live in it. If one is able to find similarities between things he/she knows and things he/she does not know (see a model), there will be no urge to reject the new thing as strange and unfamiliar. This approach can significantly accelerate the process of learning new things if founded in the forming of student's perception. Otherwise, the student should perform a sequence of observations in order to understand the matter (concept or connection). In the case of similarities or analogies, one can substitute the needed observations with ones done previously when there was the need to form concepts of the given similarities or analogies, therefore accelerating the process of comprehension. So, with the use of models and reverse thinking, we can hypothetically reduce the time needed to understand new things. Only hypothetically because there might be a situation where the student does not have an applicable area where to interpret the new object, but the practice confirms that usually this works in many cases. Reverse thinking happens in the level of images, but verbal thinking requires a fixed pattern, reducing its effectivity. Without reverse thinking a student has smaller amount of available and well understood things than with it. Normally, almost all of us use reverse thinking very often, but if this ability is not specifically trained, it is not used when encountering new things, because it seems to be too complicated.

In the education process, the biggest mistake is to teach every subject separately not demonstrating its connection with other subjects. Connection with the world one is familiar with in order to help understand new things can be formed with the help of models, but in a way it could limit one's thinking, because forms connection with only the given subject-this is restricted reverse thinking. In order to substantially widen the scope of the students they must learn to do this in various subjects as well as outside the school environment, practically train the ability to grasp new things-use reverse thinking.

\section{Reverse Thinking and Fantasy/Creativity}

Finding a model in some cases to use in another area not always indicates reverse thinking. Every new discovery, every engineer technical solution, every idea, and result formalised in a corresponding theory are done on the basis of the author's inner model which is an individual thing to each of us, but definitely connected with the area we are most familiar with, where we feel most comfortable and have the feeling that everything follows a natural pattern and cannot happen differently. Almost nobody thinks in formulas, but the inner model changes depending on the formulas. A typical case of experimental science, we have experimental data that shows how a process happens out of which we have to draw a conclusion why this happens exactly like that, we must build our inner model which will be adjusted, formalised later on.

Reverse thinking, as we see it, is to permanently create a model for each new thing, which makes us think that the things done by the author could be created/formalised by us again if we used our model. All this, of course, is true only with things invented or discovered by humans and formalised or formed into material objects.

So, reverse thinking in the sense of this paper is a permanent ability to subconsciously form inner models that can lead to a similar result as the previous author has reached. Each object that has its model (in the given area) can be added to those objects that can be further used in order to form new models. Each new model adds to the base of creative resources. Reverse thinking is an expression of fantasy, because it leads to finding/building analogous or similar objects. Further solving this process, an individual can be guided by the data of the experiment or create an artificial field in imagination/fantasy, for which a model is created and 
naturalism found-a new theory or invention. New concepts arise from new observations, but those each individual can create in his/her imagination from sets of existing concepts, by combining them in different combinations and doing different observations in imagination in this new environment. Reverse thinking eliminates the barriers between different fields and allows working freely with concepts from different fields that allow the mixing of different objects in strange designs that create new objects for new observations in imagination that can create new concepts that can be the basis of new discoveries. Mathematics and a number of other sciences were created on this basis. Practically, reverse thinking is a game of subconsciousness, which is trained consciously by creating patterns for things.

\section{The Results Obtained on the Basis of These Assumptions}

At the beginning of the joint experiment between the Latvian Education Fund and the Riga English Gymnasium, limited reverse thinking was localized to the comprehension of a specific topic in the educational process for high school. This was due to the fact that the need for reverse thinking was not yet identified and there was a need to provide knowledge as information and comprehension within a particular school and to offer it to the education system. Four average 10th grade students of Riga English Gymnasium created models in accordance with the Latvian Republic study standard for all subjects from Grades 10 to 12 in four subjects-mathematics, biology, chemistry, and physics. This process was led by teachers specially trained in using models. Students created at least $75 \%$ of the models without the help of teachers even for the subjects they had not mastered. Leading teachers have been rewarded with the Latvian Innovation Award for Education in 2011 for this training material, which has been available for nine years (see http://www.goerudio.com ${ }^{1}$ ), and this material has been further enhanced by student-based models.

A scandalous result, since everything was created by those who have not even studied that yet, so why do we have to spend so much time at school? This experiment shows that limited reverse thinking works in the context of specific topics. These students, who made the starting model base at http://www.goerudio.com, later admitted that they are still creating their own inner models without knowing it, and they feel comfortable when the task is successful. It is debatable, because the experiment was conducted in one school and the results are not accurately documented. Next, the EU project "Goerudio" was implemented to find out why students do not want to learn science subjects and how to better present them. The model method was chosen as the most usable, because it provided a comprehension. A new EU project called "GoScience" has now been launched, which is starting to expand the use of the modeling method. Reverse thinking was added as an additional contribution to the "GoScience" project, as it was needed to motivate especially talented students and make them busier with work within the project.

\section{Reverse Thinking and Recognition}

It is important to recognize things because of reading a piece of instruction, a scientific or popular science article, or encountering a new gadget or other new technology solution, without knowing the things that matter, we do not understand what is being discussed. "Known + known + unknown + known $+\ldots=$ unknown." Reverse thinking allows you to automatically involve as many fields as possible in the subconscious level of recognition process. The recognition process is accelerated and verified by the models of these fields, because

\footnotetext{
1 The website for free public use created by "EM Methodologies" Ltd. (2009) and supported by Latvian Education Foundation.
} 
the usual amount of observations is replaced by past observations, which can reduce the recognizable thing by means of models of already known things.

\section{Reverse Thinking and Comprehension}

Reverse thinking is generally based on comprehension, if there is no comprehension, then there is no reverse thinking. Comprehension in the learning process is not a simple task, as it is often unclear whether the confident explanation of a student is a previously learned or borrowed and modified text. This simplified approach is often met by teachers and trainers. Also, comprehension cannot be shown by confidently solving math problems and tasks in other subjects, as they can be solved by learning some schemes and modification techniques that do not show comprehension, but rather excellent learning skills. Comprehension can really be detected if an individual can find analogues and similarities (models) in other areas of the topic/subject. Comprehension in certain areas also does not give a full picture of things. There are qualitative characteristics that, for example, are quantifiable and have a number of other characteristics, but comprehension answers to the question "Why?". Similarly, models that show whether it has been understood or not, answer the question "Why?". A realistic continuous flow of comprehension can be obtained when models are created intuitively for everything that is coming and it is concluded known or not. Without comprehension, there is no reverse thinking. But without a reversed approach, it is hard to gain comprehension.

Reverse thinking is not an innovation, but we have purposefully specified it in our own way. First of all, it is about each student's fantasy. Each thing is reduced to a well known field where the student has an intuitive and precise idea of a thing in a well known field to him/her. A model that is intuitively natural to the student is then found or created, which is not a surprise, since everything is familiar and therefore natural. The comprehension of naturalness is comparable to the feeling of comfort, which signals that we see, know, and expect everything and there will be no surprises. This means that the student will feel protected, since he/she is not involved with anything unnatural. Otherwise, if the model is not found, there is discomfort, "I do not know/do not comprehend." There are three solutions to the do not know/do not comprehend situation:

1. Do not know/do not comprehend and cancel;

2. Do not know/do not comprehend and search what to do with it next;

3. Do not know/do not comprehend and search for enemies.

"Do not know/do not comprehend" situation often occurs in each student's space, but only few have the ability to deal with such situations unless they have the ability of reverse thinking. After that, a student with reverse thinking will be inevitably in the position of a scientist and will want to satisfy his/her intuition's demand for an answer, why this situation is happening in general, and so on. In practice, the student will try to create a model that will convince himself/herself and it would be necessary for the student to test this model so that it would not mislead him/her if it turns out to be wrong. In our understanding, reverse thinking is the perpetual ability to create models mostly in the subconscious in order to obtain a sense of comfort-a comprehension of a particular thing (concept or relation).

\section{Learning Reverse Thinking}

Reverse thinking cannot be obtained by learning it as a school subject. For the most talented students (it can be more difficult for the less talented and they are most likely to gain limited reverse thinking), it is best achieved through a specially designed learning process at all levels of education from pre-school to university. 
The key to teaching each subject is to show that it is possible to see its analogues/similarities in other familiar fields, to notice that in many cases things are similar-they fit the same model, and to constantly develop this ability. Modeling requires students to have a comprehensive approach that goes far beyond the scope of the topic, and thus, gains the ability to locate/see analogies/similarities in topics that are different in the fields that they already know. Modeling method and generated models are verifiable, easier to distribute and accumulate, and publicly available, which demonstrates the positive features of this approach.

Developing and using models in the learning process is a developed and tested methodology, but it only applies to the comprehension of specific STEM topics, not for the permanent use for everyday life and other new things. It is important to add other things from everyday life to the specific topics of STEM subjects, which can show that you can obtain things and specific information also outside the education standards of STEM subjects. This approach will create the need to "dig" into the instructions, descriptions, and other information that will give the student the opportunity to develop his/her own internal model and discuss it with the audience. The discussion of student models can only happen within the context of correctness in a specific topic, because any other objections do not fit here by definition, since each student forms the most acceptable model for him/her that allows them to intuitively feel the nature of the subject. This is an essential part of the learning process and the teacher has to pay special attention to this. They might not like the model, but if it is correct, it cannot be criticized. Learning reverse thinking is not a short-term process. It can be achieved with a long-term training and is complemented by the methodology of modeling and model use.

Reverse thinking does not form within one subject. If the model method is used in only one subject, the result is an impression that it only fits this subject (limited reverse thinking), therefore it is necessary to ensure the use of models in several subjects at the same time.

Using this method in the environment of many subjects, the student becomes aware of the comprehensive use of the method and it makes it easier to simulate many things that are not relevant to these subjects.

The abovementioned results can only be achieved if there are teachers who use this method. Educated teachers are the most important part for a successful implementation of this method. It would be very good if they would follow a succession of a teacher-school-university. Of course, this does not mean that the method can be only used in schools. Reverse thinking gives the student the perception and ability to use the same model for a number of subjects, which also reveals the similarities of many fields.

In order to get closer to reverse thinking, the learning process could be transformed as follows: The teacher instructs to create a model for a certain term and the student creates the model. Another teacher in another subject instructs to create a model for a topic and the student can adequately use the same model, but in a different subject. This can show the student that the same model can fit multiple concepts at the same time. Such an approach requires a great deal of synergy between teachers. It is often the case that one subject teaches something that can be used in another, such as physics and mathematics.

The more subjects, the more opportunities to practice. Practice of creation and usage of the model in a given subject and models in other subjects and demonstration how this approach is used universally and intentionally show that this approach lets you understand a lot of new things, gradually habituates to unintentional use of the models, finally leading to subconscious use of them.

A methodology was designed for the use of models (Gaidule \& Heidingers, 2015), but the scheme for promoting reverse thinking described in the publication. "Preparation stage-creation of model-improvement of 
model-assessement of model" may be supplemented by model verification. Does it answer the question "Why?" and adapt the worksheets accordingly? The methodology is supplemented by working on the development of the skills of especially talented students, where it is useful to instruct to build a model after the results of the experiment, in order to answer the question "Why is it happening?" instead of "How does it happen?".

\section{Conclusions}

In the learning process, the focus should be on answering the question "Why?" rather than "How?", which is best demonstrated by models.

Reverse thinking is not possible without building models in several subjects at the same time and for a long time.

A large amount of students will only have limited reverse thinking due to their own lack of fantasy. Not everyone has enough fantasy and it is a fact that has to be accepted, that there are especially talented students, but not all. Limited reverse thinking is also an achievement, as it significantly reduces the possibility of mistakes in certain fields and discomfort arises with incorrect solutions.

There should be a sufficient amount of model examples created by students, in order to encourage other students to create such models. There should be teachers who know how to use models in the learning process in accordance with the updated methodology. Model method should be used in several subjects at the same time.

\section{References}

Cyriel, K. (2017). Retrieved from https://www.cyrielkortleven.com/reverse-thinking/

Gaidule, A., \& Heidingers, U. (2015). The use of associative images (models) for the development of comprehension in sciences education. American Journal of Educational Research, 3(10), 1305-1310. doi:10.12691/education-3-10-15

Vitkovskis, R., Heidingers, U., Jakubova, I., Rikmane, I., \& Krišmane A. (2012). Method and tool to achieve necessary level of comprehension. US-China Education Rewiev A, 2(8), 727-732. 